\title{
Search for new flat-spectrum radio sources
}

\author{
H. Teräsranta ${ }^{1}$, S. Urpo ${ }^{1}$, S. Wiren ${ }^{2}$, and M. Valtonen ${ }^{2}$ \\ 1 Metsähovi radio research station, Helsinki University of Technology, 02540 Kylmälä, Finland \\ 2 Tuorla Observatory, 21500 Piikkiö, Finland
}

Received 15 November 2000 / Accepted 19 December 2000

\begin{abstract}
GHz}$ continuum observations with the Metsähovi Radio telescope are used to identifying new flat spectrum radio sources selected from lower frequency survey data. The sample is complete to sources with a flux higher than $0.5 \mathrm{Jy}$ at $1.4 \mathrm{GHz}, \alpha \geq-0.5$ between 1.4 and $4.85 \mathrm{GHz}$, declination from 0 to +75 degrees and sources more than $20^{\circ}$ from the galactic plane. Additional strong sources from the $5 \mathrm{GHz}$ survey were observed to get the sample as complete as possible down to $1.0 \mathrm{Jy}$ at $22 \mathrm{GHz}$ for extrapolation of source counts to lower flux levels. The new flat spectrum sources will be added to our monitoring sample and their duty cycle will be studied for the coming $\gamma$-ray observatiories AGILE and GLAST.
\end{abstract}

Key words. galaxies: active - BL Lacertae objects: general - quasars: general - radio continuum: galaxies - radio continuum: general

\section{Introduction}

One common feature in the gamma-ray detected AGN and sources strong enough to be notable foreground sources over a broad band for the PLANCK cosmic background surveyer, operating from 30 to $860 \mathrm{GHz}$, is a flat spectrum from $\mathrm{cm}$ to $\mathrm{mm}$ wavebands. Many of those flat-spectrum sources are still unknown, as the highest all-sky survey frequency is now $5 \mathrm{GHz}$. One could try to extrapolate the spectral behaviour of AGN from lower frequency source surveys, for example 1.4 and $4.85 \mathrm{GHz}$, but such generalization is possible only for some classes of sources, like regular radio galaxies, whose spectral index remains the same over a wide frequency range. A better solution for finding flat spectrum sources would be to do a complete survey at a higher frequency, around $20-40 \mathrm{GHz}$ down to 20-30 mJy levels. The variability of flat spectrum sources would result in a considerable part of the sources being in a quiet stage and thus misidentified as deep spectrum sources, when compared to other frequency surveys done at different epochs. Doing the high frequency survey twice should show the sources in a more active phase at least during the other observation.

A large sample of sources (458) with a flux greater than $0.5 \mathrm{Jy}$ at $1.4 \mathrm{GHz}$ and spectral index $\alpha \geq-0.5$ between 1.4 and $4.85 \mathrm{GHz}$ has been observed with the Metsähovi radio telescope at $22 \mathrm{GHz}$ to find new flat spectrum sources. A similar sample has been observed at $8.4 \mathrm{GHz}$ with the VLA, Patnaik et al. (1992); Browne et al. (1998);

Send offprint requests to: H. Teräsranta,

e-mail: hte@alpha.hut.fi
Wilkinson et al. (1998), to find interferometer phase calibration sources (the Jodrell Bank - VLA Astrometric Survey, in short JVAS). Their sample consisted of Northern sources with $\alpha \geq-0.4$ between 1.4 and $4.85 \mathrm{GHz}$ with a flux density greater than $0.2 \mathrm{Jy}$ at $4.85 \mathrm{GHz}$. Their $4.85 \mathrm{GHz}$ fluxes differ slightly from the GB6 cataloque, as they have done their own fitting to the original maps. Nearly all of the sources in our study are included in the JVAS survey; the missing objects are mainly in the steep spectrum sources.

In a study of the spectra from 1 to $22 \mathrm{GHz}$ of 550 radio sources with the RATAN-antenna, Kovalev et al. (1999), 195 sources (exluding our long term monitoring sources) are common with this study, although their selection criteria are declinations between -30 and +43 degrees and the correlated VLBI flux at $13 \mathrm{~cm}$ being higher than $0.1 \mathrm{Jy}$. In general the flux densities at $22 \mathrm{GHz}$ in Kovalev et al. (1999) and this study are for the common sources quite similar, though the single epoch observations are more than a year apart. In most cases the sensitivity of the RATAN-survey is better than surveys with the Metsähovi antenna.

In our study special attention has been given to sources with a steeper spectral index in the lower frequency part to find what portion of them are in reality flat spectrum sources in the mm-wave band. The reason why these sources have a rather low spectral index in the cataloques at low frequencies could be due to the different epcochs when the surveys are made. Even if the source is observed simultaneously at both survey frequencies, the source can be in an outburst phase, which could turn the spectra to 
inverted if in the rising phase, or to steeper spectrum if it was in the declining phase, if we assume the normal scenario where the outburst moves from higher frequencies to lower ones. For variable flat spectrum sources, giving a spectral index between two single epoch observations might be enough for statistical studies of large samples, but when looking at individual sources, wrong conclusions can be drawn from the source being in a different phase of outburst evolution. The variability amplitudes are smaller at lower frequencies, like 1.4 and $4.85 \mathrm{GHz}$ and thus the problem is more serious at $\mathrm{mm}$ and submillimeter bands. A sub-group in this sample are the $\gamma$-detected AGN, which are all flat spectrum sources in the cm-mm wavebands. The newfound flat-spectrum sources will be added to the Metsähovi monitoring sample for observing their duty cycle before the launch of the more sensitive $\gamma$-ray laboratories AGILE and GLAST.

\section{Observations}

The observations presented here have been done with the Metsähovi radio telescope. The selection criteria for the sources has been a source declination between 0 and +75 degrees, galactic distance $|b| \geq 20^{\circ}$, flux density higher or equal of $0.5 \mathrm{Jy}$ at $1.4 \mathrm{GHz}$ and a spectral index $\alpha$ being higher than -0.6 between 1.4 and $4.85 \mathrm{GHz}$ as given in White \& Becker (1992). The $20^{\circ}$ limit from the galactic plane comes from the observing strategy of the cosmic background observer PLANCK. Positive $\alpha$ is considered a rising or inverted spectra in this study $\left(S_{\nu} \propto \nu^{\alpha}\right)$.

The Green Bank beam size at $1.4 \mathrm{GHz}$ is in the order of $10^{\prime}$ and $3.5^{\prime}$ at $4.85 \mathrm{GHz}$ compared to the Metsähovi antennas beam of $4^{\prime}$ at $22 \mathrm{GHz}$. Sources marked as extended in the $1.4 \mathrm{GHz}$ cataloque have been included if their spectral index is given, which means that their $4.85 \mathrm{GHz}$ flux is given in the GB6 cataloque. The extended structures in radio sources tend to have a steeper spectrum and thus their impact to the higher frequency total flux should be much smaller than at lower frequencies (a few GHz). The low sensitivity of our system, resulting mostly from the rather small radome inclosed antenna $(D=13.7 \mathrm{~m})$, together with the modest angular resolution does not allow us to map the sources or even try to estimate the intensity profiles. The number of extended sources in the sample was 23 . From the 23 extended sources three $0836+710$, $0954+658$ and $1222+216$ have shown gamma radiation, Hartman et al. (1999). As the fraction of these gammaemitting sources is over $10 \%$ from the sources classified as extended, the sources showing extended structure at low frequencies should not be flagged out if looking for flat spectrum or gamma-ray emitting sources. Besides the sample described above, stronger sources $(S>1.0 \mathrm{Jy}$ at $5 \mathrm{GHz}$ ) were also observed to get the population of sources whose flux on average exeeds $1 \mathrm{Jy}$ at $22 \mathrm{GHz}$ as complete as possible. Many of these additional sources are regular radio galaxies with a steep spectra $(\alpha \leq-0.7)$.

As many of these sources are variable in nature and some of them have been monitored with our telescope for nearly two decades, we estimate a mean value for the flux at $22 \mathrm{GHz}$ in the following manner: first the fluxes of individual sources are averaged weekly and from those values a mean value and its error estimate is calculated. The weekly mean value is to soften the effects for our non evenly sampled monitoring, as the sampling tends to be quite dense during observing campaings for specific sources, as well as during ultimate events, like large outbursts. The monitoring data was used until May 2000. Most of the single epoch observations were done between October 1998 and October 2000. The observing procedures and data reduction for obtaining the fluxes are presented in full in Teräsranta et al. (1998). Part of the sample was observed only twice during 1988, Wiren et al. (1992). The majority of the sources were observed only once which leads to the question: can their observed flux be used as a representative value for the mean value? If we assume that the variable sources have a similar duty-cycle, then observing a large sample of them would result in sources being in random phases of outburst, which would on average give a value similar to taking a mean value of a completely sampled flux curve. Sources which are in a very quiet stage for long periodes and randomly show strong outbursts, like III ZW 2 and $0109+224$ can be missed easily in a single epoch observation and thus misidentified, when looking for stronger flat spectrum sources.

Prior the year 1993 the sensitivity of our $22 \mathrm{GHz}$ receiver was only about $850 \mathrm{~K} \mathrm{DSB}$, compared to the later versions $300 \mathrm{~K}$ DSB. This leads to the fact that the error margins in the earlier observations are considerably larger than presently. In some cases the poor $S / N$ observations during 1988 were replaced by more sensitive ones with the current receiver. The rather low sensitivity of our receiving system leads to the fact that we are not able to detect most of the steep spectrum sources. If we had a source in the lower edge of our criteria: a flux of $0.5 \mathrm{Jy}$ at $1.4 \mathrm{GHz}$ and a spectral index -0.5 continuing all the way to $22 \mathrm{GHz}$, we would have a flux of only $0.095 \mathrm{Jy}$ at $22 \mathrm{GHz}$, which would remain undetected even if the error was the lowest we have recorded for these single epoch observations (0.02 Jy).

\section{Results}

The flux densities obtained at $22 \mathrm{GHz}$ and also the spectral index $\alpha_{2}$ calculated against the $4.85 \mathrm{GHz}$ fluxes from the Green Bank survey, Gregory et al. (1996), are presented in Table 1. The lower frequency spectral index $\alpha_{1}$ (between 4.85 and $1.4 \mathrm{GHz}$ ) is also given. The sources detected with EGRET in the $\gamma$-ray band are marked with an asterix $(*)$. The number of observations states the number of weeks on which the source has been observed. The criteria for a detection has been set to $5 \sigma$ and spectral indexes are presented only for the detected sources. The error estimates for single epoch observations have been in their lowest in the order of $20 \mathrm{mJy}$ and highest around $100 \mathrm{mJy}$. It is clear that during colder seasons the receiving system noise temperatures are lower, but due to the 
Table 1. The sample for search of flat spectrum sources: B1950 designation (an asterix $*$ marking sources detected at $\gamma$-rays according to Hartman et al. 1999), observed flux density at $22 \mathrm{GHz}, 1 \sigma$ error estimate, number of observations, flux at $4.85 \mathrm{GHz}$ from Gregory et al. (1996), $\alpha_{4.85,22}$ and $\alpha_{1.4,4.85}$ from White \& Becker (1992)

\begin{tabular}{|c|c|c|c|c|c|c|}
\hline Source & $S(22)$ & $\sigma$ & $n$ & $S(5)$ & $\alpha_{2}$ & $\alpha_{1}$ \\
\hline $0003+380$ & 0.43 & 0.07 & 1 & 0.568 & -0.18 & -0.1 \\
\hline $0006+397$ & 0.30 & 0.05 & 1 & 0.338 & -0.08 & -0.4 \\
\hline $0007+171$ & 0.74 & 0.11 & 1 & 0.989 & -0.19 & 0.1 \\
\hline $0009+081$ & 0.56 & 0.10 & 1 & 0.400 & 0.22 & -0.2 \\
\hline $0010+405$ & 0.60 & 0.10 & 4 & 1.035 & -0.36 & -0.4 \\
\hline $0017+257$ & 0.36 & 0.04 & 1 & 0.435 & -0.13 & -0.4 \\
\hline $0017+200$ & 0.65 & 0.10 & 1 & 0.710 & -0.06 & 0.2 \\
\hline $0022+390$ & 0.28 & 0.05 & 1 & 0.712 & -0.27 & -0.1 \\
\hline $0024+348$ & 0.10 & 0.03 & 1 & 0.463 & & -0.5 \\
\hline $0026+346$ & 0.64 & 0.08 & 2 & 1.182 & -0.18 & -0.2 \\
\hline $0030+196$ & 0.23 & 0.07 & 1 & 0.997 & & -0.5 \\
\hline $0032+276$ & 0.24 & 0.06 & 1 & 0.576 & & -0.4 \\
\hline $0033+098$ & 0.33 & 0.06 & 1 & 0.377 & -0.09 & -0.5 \\
\hline $0035+367$ & 0.09 & 0.06 & 1 & 0.457 & & -0.5 \\
\hline $0035+121$ & 0.26 & 0.09 & 1 & 0.665 & & -0.3 \\
\hline $0035+413$ & 0.51 & 0.03 & 1 & 1.144 & -0.23 & 0.7 \\
\hline $0039+230$ & 0.48 & 0.04 & 1 & 1.604 & -0.34 & 0.5 \\
\hline $0040+172$ & 0.10 & 0.05 & 1 & 0.313 & & -0.5 \\
\hline $0055+300$ & 0.81 & 0.09 & 2 & 0.914 & -0.03 & -0.2 \\
\hline $0104+231$ & 0.08 & 0.07 & 1 & 0.268 & & -0.5 \\
\hline $0106+013$ & 2.19 & 0.07 & 124 & 4.180 & -0.18 & -0.1 \\
\hline $0106+130$ & 1.31 & 0.13 & 1 & 3.691 & -0.69 & -1.0 \\
\hline $0108+388$ & 0.28 & 0.02 & 2 & 1.340 & -0.45 & 0.9 \\
\hline $0109+224$ & 1.04 & 0.05 & 110 & 0.304 & 0.81 & -0.1 \\
\hline $0111+021$ & 0.71 & 0.10 & 1 & 0.608 & 0.04 & 0.2 \\
\hline $0113+154$ & 0.05 & 0.07 & 1 & 0.412 & & -0.5 \\
\hline $0114+074$ & 0.20 & 0.03 & 1 & 0.866 & -0.97 & -0.4 \\
\hline $0116+319$ & 0.56 & 0.08 & 4 & 1.609 & -0.70 & -0.5 \\
\hline $0119+115$ & 1.19 & 0.09 & 4 & 1.126 & 0.02 & -0.1 \\
\hline $0119+041 *$ & 1.56 & 0.02 & 2 & 1.235 & 0.07 & 0.2 \\
\hline $0119+247$ & 0.78 & 0.06 & 1 & 0.764 & 0.01 & 0.2 \\
\hline $0123+257$ & 0.57 & 0.04 & 1 & 1.303 & -0.24 & 0.4 \\
\hline $0124+189$ & 0.20 & 0.07 & 1 & 0.671 & & -0.5 \\
\hline $0127+145$ & 0.25 & 0.10 & 1 & 0.570 & & -0.2 \\
\hline $0134+329$ & 1.14 & 0.06 & 1 & 5.718 & -1.06 & -0.8 \\
\hline $0137+012$ & 0.34 & 0.03 & 2 & 0.665 & -0.44 & -0.5 \\
\hline $0144+209$ & -0.01 & 0.07 & 1 & 0.600 & & -0.5 \\
\hline $0146+056$ & 1.04 & 0.04 & 1 & 1.473 & -0.10 & 0.6 \\
\hline $0148+274$ & 0.37 & 0.06 & 1 & 0.995 & -0.28 & 0.4 \\
\hline $0149+218$ & 1.27 & 0.02 & 95 & 1.073 & 0.05 & -0.2 \\
\hline $0149+335$ & 0.13 & 0.06 & 1 & 0.611 & & -0.4 \\
\hline $0159+034$ & 0.35 & 0.04 & 1 & 0.312 & 0.08 & -0.4 \\
\hline $0201+365$ & 0.09 & 0.07 & 1 & 0.358 & & -0.4 \\
\hline $0201+113$ & 0.55 & 0.07 & 1 & 0.824 & -0.12 & -0.2 \\
\hline $0202+319$ & 0.82 & 0.16 & 4 & 0.934 & -0.04 & -0.1 \\
\hline $0202+149 *$ & 2.32 & 0.06 & 127 & 3.073 & -0.19 & -0.4 \\
\hline $0204+316$ & 0.06 & 0.07 & 1 & 0.429 & & -0.4 \\
\hline $0212+171$ & 0.14 & 0.03 & 1 & 0.294 & & -0.5 \\
\hline $0215+015$ & 1.39 & 0.27 & 4 & 1.419 & -0.01 & 0.8 \\
\hline $0217+367$ & 0.13 & 0.08 & 1 & 0.184 & & -0.5 \\
\hline $0217+324$ & 0.24 & 0.04 & 1 & 0.647 & -0.66 & -0.4 \\
\hline $0218+357$ & 1.01 & 0.03 & 41 & 1.480 & -0.11 & 0.0 \\
\hline $0221+067$ & 1.36 & 0.25 & 4 & 0.989 & 0.09 & 0.0 \\
\hline
\end{tabular}

Table 1. continued

\begin{tabular}{|c|c|c|c|c|c|c|}
\hline Source & $S(22)$ & $\sigma$ & $n$ & $S(5)$ & $\alpha_{2}$ & $\alpha_{1}$ \\
\hline $0223+341$ & 0.74 & 0.02 & 2 & 1.629 & -0.52 & -0.4 \\
\hline $0226+086$ & 0.22 & 0.08 & 1 & 0.329 & & -0.5 \\
\hline $0229+131$ & 0.95 & 0.04 & 22 & 2.608 & -0.29 & 0.4 \\
\hline $0229+262$ & 0.47 & 0.05 & 1 & 0.435 & 0.02 & -0.1 \\
\hline $0234+285 *$ & 2.61 & 0.09 & 92 & 2.794 & -0.02 & 0.3 \\
\hline $0235+164 *$ & 2.12 & 0.09 & 190 & 1.935 & 0.03 & -0.1 \\
\hline $0235+090$ & 0.20 & 0.04 & 1 & 0.533 & -0.65 & -0.4 \\
\hline $0237+040$ & 0.58 & 0.03 & 1 & 1.061 & -0.17 & 0.4 \\
\hline $0239+108$ & 0.85 & 0.07 & 1 & 1.473 & -0.16 & -0.1 \\
\hline $0253+133$ & 0.22 & 0.07 & 1 & 0.499 & & 0.0 \\
\hline $0256+075$ & 0.64 & 0.19 & 2 & 0.648 & 0.00 & 0.0 \\
\hline $0258+058$ & 0.21 & 0.04 & 1 & 0.321 & -0.12 & -0.3 \\
\hline $0259+121$ & 0.18 & 0.07 & 1 & 0.473 & & -0.2 \\
\hline $0301+336$ & 0.62 & 0.10 & 1 & 0.706 & -0.04 & -0.3 \\
\hline $0306+103$ & 0.89 & 0.03 & 38 & 0.597 & 0.11 & 0.0 \\
\hline $0319+121$ & 0.81 & 0.03 & 1 & 1.543 & -0.18 & -0.1 \\
\hline $0322+222$ & 0.85 & 0.05 & 1 & 0.817 & 0.01 & 0.2 \\
\hline $0326+277$ & 0.32 & 0.04 & 1 & 0.732 & -0.55 & -0.4 \\
\hline $0338+074$ & 0.25 & 0.09 & 1 & 0.536 & & -0.5 \\
\hline $0348+049$ & 0.13 & 0.03 & 1 & 0.482 & & -0.4 \\
\hline $0351+045$ & 0.17 & 0.02 & 1 & 0.444 & -0.27 & -0.2 \\
\hline $0406+121$ & 0.37 & 0.10 & 1 & 0.843 & & 0.2 \\
\hline $0409+229$ & 0.46 & 0.08 & 1 & 0.925 & -0.20 & -0.2 \\
\hline $0421+145$ & 0.26 & 0.08 & 1 & 0.318 & & -0.3 \\
\hline $0421+019$ & 0.35 & 0.03 & 1 & 0.711 & -0.47 & -0.4 \\
\hline $0422+004$ & 0.68 & 0.03 & 91 & 0.879 & -0.07 & 0.6 \\
\hline $0423+051$ & 0.42 & 0.05 & 1 & 0.590 & -0.10 & -0.1 \\
\hline $0425+048$ & 0.35 & 0.09 & 1 & 0.700 & & -0.3 \\
\hline $0425+094$ & 0.10 & & 1 & 0.276 & & -0.5 \\
\hline $0430+052$ & 3.10 & 0.06 & 197 & 4.198 & -0.09 & -0.1 \\
\hline $0441+106$ & 0.14 & 0.04 & 1 & 0.403 & & -0.4 \\
\hline $0445+097$ & 0.23 & 0.07 & 1 & 0.580 & & -0.5 \\
\hline $0446+112 *$ & 1.29 & 0.06 & 65 & 0.794 & 0.14 & -0.1 \\
\hline $0454+066$ & 0.50 & 0.06 & 1 & 0.623 & -0.06 & -0.2 \\
\hline $0457+024$ & 0.47 & 0.03 & 1 & 1.782 & -0.38 & 0.0 \\
\hline $0459+060 *$ & 0.50 & 0.04 & 1 & 0.874 & -0.16 & -0.1 \\
\hline $0500+019$ & 0.80 & 0.04 & 2 & 1.937 & -0.25 & -0.1 \\
\hline $0502+049$ & & 0.03 & 1 & & -0.14 & 0.3 \\
\hline $0604+728$ & 0.17 & 0.04 & 1 & 0.664 & & -0.2 \\
\hline $0633+734$ & 0.23 & 0.04 & 1 & 0.711 & -0.32 & -0.2 \\
\hline $0700+470$ & 0.17 & 0.06 & 1 & 0.465 & & -0.5 \\
\hline $0707+476$ & 0.84 & 0.18 & 9 & 0.984 & -0.05 & -0.1 \\
\hline $0710+439$ & 0.78 & 0.11 & 1 & 1.607 & -0.21 & -0.1 \\
\hline $0716+714 *$ & 1.04 & 0.06 & 81 & 0.859 & 0.05 & 0.0 \\
\hline $0733+597$ & 0.02 & 0.07 & 1 & 0.370 & & -0.4 \\
\hline $0738+313$ & 1.22 & 0.12 & 5 & 2.623 & -0.22 & 0.2 \\
\hline $0738+272$ & 0.36 & 0.06 & 1 & 0.460 & -0.16 & -0.5 \\
\hline $0739+398$ & 0.22 & 0.04 & 1 & 0.416 & -0.42 & -0.5 \\
\hline $0745+241$ & 1.05 & 0.04 & 2 & 1.263 & -0.05 & 0.2 \\
\hline $0746+483$ & 0.63 & 0.11 & 1 & 0.902 & -0.10 & 0.2 \\
\hline $0748+333$ & 0.29 & 0.03 & 1 & 0.444 & -0.28 & -0.4 \\
\hline $0749+540$ & 1.60 & 0.11 & 1 & 0.964 & 0.14 & 0.1 \\
\hline $0749+426$ & 0.12 & 0.10 & 1 & 0.450 & & -0.3 \\
\hline $0759+183$ & 0.52 & 0.10 & 1 & 0.457 & 0.04 & -0.2 \\
\hline $0802+212$ & 0.22 & 0.03 & 1 & 0.551 & -0.61 & -0.4 \\
\hline $0804+499 *$ & 1.29 & 0.05 & 132 & 1.315 & -0.01 & 0.3 \\
\hline $0805+269$ & 0.12 & 0.08 & 1 & 0.263 & & -0.5 \\
\hline $0809+483$ & 0.75 & 0.07 & 1 & 4.504 & -1.19 & -0.9 \\
\hline
\end{tabular}


Table 1. continued

\begin{tabular}{|c|c|c|c|c|c|c|}
\hline Source & $S(22)$ & $\sigma$ & $n$ & $S(5)$ & $\alpha_{2}$ & $\alpha_{1}$ \\
\hline $0812+367$ & 0.69 & 0.13 & 1 & 0.994 & -0.10 & 0.0 \\
\hline $0814+326$ & 0.27 & 0.04 & 1 & 0.581 & -0.22 & 0.0 \\
\hline $0814+425$ & 1.08 & 0.04 & 107 & 1.866 & -0.16 & 0.0 \\
\hline $0820+560$ & 1.03 & 0.06 & 1 & 1.155 & -0.03 & -0.1 \\
\hline $0820+225$ & 0.81 & 0.02 & 29 & 1.392 & -0.15 & -0.3 \\
\hline $0821+621$ & 0.25 & 0.06 & 1 & 0.619 & & 0.0 \\
\hline $0821+394$ & 1.38 & 0.11 & 1 & 1.031 & 0.08 & -0.3 \\
\hline $0823+033$ & 1.63 & 0.18 & 6 & 1.354 & 0.05 & 0.0 \\
\hline $0824+355$ & 0.48 & 0.05 & 1 & 0.751 & -0.13 & -0.1 \\
\hline $0827+243 *$ & 1.54 & 0.04 & 66 & 0.886 & 0.16 & 0.0 \\
\hline $0829+187$ & 0.30 & 0.07 & 1 & 0.707 & & -0.3 \\
\hline $0829+046 *$ & 1.11 & 0.09 & 18 & 1.913 & -0.16 & 0.7 \\
\hline $0831+557$ & 0.84 & 0.02 & 2 & 5.740 & -0.55 & -0.2 \\
\hline $0833+585$ & 0.65 & 0.08 & 1 & 0.717 & -0.03 & 0.1 \\
\hline $0834+250$ & 0.53 & 0.05 & 1 & 0.458 & 0.04 & -0.1 \\
\hline $0836+710 *$ & 1.89 & 0.04 & 84 & 2.342 & -0.14 & -0.4 \\
\hline $0837+035$ & 0.35 & 0.05 & 1 & 0.673 & -0.19 & -0.1 \\
\hline $0839+187$ & 0.64 & 0.07 & 2 & 0.898 & -0.10 & -0.2 \\
\hline $0847+379$ & 0.29 & 0.02 & 1 & 0.391 & -0.20 & -0.4 \\
\hline $0850+581$ & 0.51 & 0.05 & 10 & 1.184 & -0.24 & -0.1 \\
\hline $0851+202 *$ & 3.58 & 0.13 & 263 & 2.908 & 0.06 & 0.1 \\
\hline $0851+071$ & 0.32 & 0.04 & 1 & 0.489 & -0.28 & -0.4 \\
\hline $0859+681$ & 0.29 & 0.05 & 1 & 0.661 & -0.24 & 0.2 \\
\hline $0859+470$ & 0.81 & 0.05 & 17 & 1.264 & -0.29 & -0.5 \\
\hline $0900+428$ & 0.41 & 0.04 & 1 & 0.747 & -0.40 & -0.5 \\
\hline $0902+490$ & 0.30 & 0.10 & 1 & 0.553 & & -0.1 \\
\hline $0906+015$ & 0.81 & 0.08 & 5 & 0.896 & -0.03 & 0.0 \\
\hline $0906+430$ & 1.00 & 0.02 & 43 & 1.607 & -0.14 & -0.7 \\
\hline $0912+029$ & 0.61 & 0.05 & 1 & 0.893 & -0.11 & 0.0 \\
\hline $0913+391$ & 0.31 & 0.08 & 1 & 0.550 & & -0.5 \\
\hline $0915+099$ & 0.16 & 0.04 & 1 & 0.384 & & -0.5 \\
\hline $0917+624$ & 1.14 & 0.18 & 3 & 1.226 & -0.02 & 0.0 \\
\hline $0917+449$ & 1.18 & 0.05 & 1 & 1.085 & 0.02 & 0.2 \\
\hline $0922+005$ & 0.37 & 0.05 & 1 & 0.810 & -0.22 & 0.0 \\
\hline $0923+392$ & 9.00 & 0.17 & 238 & 6.913 & 0.08 & 0.8 \\
\hline $0928+653$ & 0.02 & 0.07 & 1 & 0.298 & & -0.5 \\
\hline $0929+533$ & 0.25 & 0.09 & 1 & 0.388 & & -0.3 \\
\hline $0930+493$ & 0.23 & 0.10 & 1 & 0.527 & & -0.2 \\
\hline $0945+408$ & 1.50 & 0.03 & 51 & 1.801 & -0.05 & 0.1 \\
\hline $0945+664$ & 0.17 & 0.04 & 1 & 1.248 & & -0.4 \\
\hline $0950+748$ & 0.32 & 0.05 & 1 & 0.778 & -0.59 & -0.4 \\
\hline $0951+699$ & 1.29 & 0.07 & 1 & 3.796 & -0.71 & -0.6 \\
\hline $0952+179$ & 0.62 & 0.08 & 1 & 0.865 & -0.10 & -0.3 \\
\hline $0953+254$ & 1.31 & 0.02 & 95 & 1.042 & 0.07 & 0.4 \\
\hline $0954+556 *$ & 1.08 & 0.02 & 62 & 2.015 & -0.18 & -0.2 \\
\hline $0954+658 *$ & 0.69 & 0.04 & 75 & 1.125 & -0.14 & 0.6 \\
\hline $0955+326$ & 0.71 & 0.05 & 1 & 0.706 & 0.00 & -0.5 \\
\hline $0955+476$ & 0.70 & 0.04 & 1 & 1.005 & -0.10 & 0.2 \\
\hline $1005+141$ & 0.62 & 0.07 & 1 & 0.841 & -0.09 & -0.2 \\
\hline $1007+062$ & 0.08 & 0.05 & 1 & 0.296 & & -0.5 \\
\hline $1007+417$ & 0.31 & 0.09 & 1 & 0.854 & & -0.5 \\
\hline $1009+067$ & 0.14 & 0.04 & 1 & 0.300 & & -0.4 \\
\hline $1010+287$ & 0.10 & 0.04 & 1 & 0.323 & & -0.5 \\
\hline $1012+232$ & 0.77 & 0.04 & 1 & 1.093 & -0.10 & -0.1 \\
\hline $1013+208$ & 0.48 & 0.04 & 1 & 0.986 & -0.21 & 0.2 \\
\hline $1015+359$ & 0.65 & 0.03 & 1 & 0.707 & -0.06 & -0.4 \\
\hline
\end{tabular}

Table 1. continued

\begin{tabular}{|c|c|c|c|c|c|c|}
\hline Source & $S(22)$ & $\sigma$ & $n$ & $S(5)$ & $\alpha_{2}$ & $\alpha_{1}$ \\
\hline $1015+491$ & 0.25 & 0.06 & 1 & 0.282 & & -0.5 \\
\hline $1016+573$ & -0.11 & 0.06 & 1 & 0.418 & & -0.5 \\
\hline $1020+400$ & 0.78 & 0.10 & 1 & 0.789 & 0.00 & -0.3 \\
\hline $1022+194$ & 0.90 & 0.13 & 1 & 0.764 & 0.05 & 0.1 \\
\hline $1030+611$ & 0.55 & 0.03 & 1 & 0.532 & 0.01 & -0.2 \\
\hline $1030+415$ & 0.79 & 0.08 & 1 & 0.439 & 0.17 & -0.3 \\
\hline $1031+567$ & 0.32 & 0.06 & 1 & 1.200 & -0.38 & -0.3 \\
\hline $1038+528$ & 0.65 & 0.05 & 15 & 0.701 & -0.02 & 0.0 \\
\hline $1038+064$ & 1.70 & 0.05 & 1 & 1.331 & 0.16 & 0.0 \\
\hline $1040+123$ & 0.71 & 0.03 & 26 & 1.759 & -0.60 & -0.6 \\
\hline $1041+536$ & 0.05 & 0.05 & 1 & 0.437 & & -0.1 \\
\hline $1042+071$ & 0.31 & 0.08 & 1 & 0.432 & & -0.2 \\
\hline $1042+178$ & 0.75 & 0.03 & 1 & 0.391 & 0.43 & -0.2 \\
\hline $1044+719$ & 0.98 & 0.08 & 1 & 1.900 & -0.44 & 1.1 \\
\hline $1048+347$ & 0.11 & 0.02 & 1 & 0.312 & -0.69 & -0.5 \\
\hline $1049+215$ & 1.14 & 0.03 & 38 & 1.076 & 0.04 & 0.1 \\
\hline $1053+704$ & 0.81 & 0.06 & 1 & 0.539 & 0.27 & 0.1 \\
\hline $1055+018$ & 3.68 & 0.07 & 142 & 3.403 & 0.05 & 0.2 \\
\hline $1055+201$ & 0.54 & 0.04 & 1 & 1.505 & -0.29 & -0.3 \\
\hline $1058+629$ & 0.09 & 0.04 & 2 & 0.693 & & 0.1 \\
\hline $1058+726$ & 0.82 & 0.10 & 1 & 0.958 & -0.01 & -0.3 \\
\hline $1100+223$ & 0.15 & 0.05 & 1 & 0.535 & & 0.1 \\
\hline $1101+384 *$ & 0.61 & 0.01 & 117 & 0.723 & -0.11 & -0.1 \\
\hline $1104+167$ & 0.27 & 0.02 & 1 & 0.561 & -0.48 & -0.3 \\
\hline $1106+023$ & 0.29 & 0.04 & 1 & 0.584 & -0.46 & -0.3 \\
\hline $1108+201$ & 0.21 & 0.03 & 1 & 0.666 & -0.76 & -0.5 \\
\hline $1111+149$ & 0.38 & 0.09 & 1 & 0.495 & & 0.0 \\
\hline $1116+128$ & 1.11 & 0.05 & 1 & 1.820 & -0.33 & 0.0 \\
\hline $1118+000$ & 0.03 & 0.04 & 1 & 0.351 & & -0.3 \\
\hline $1119+183$ & 0.39 & 0.04 & 1 & 0.744 & -0.43 & 0.1 \\
\hline $1123+264$ & 0.65 & 0.04 & 1 & 1.176 & -0.39 & 0.3 \\
\hline $1124+571$ & 0.05 & 0.05 & 2 & 0.448 & & -0.2 \\
\hline $1126+101$ & 0.14 & 0.04 & 1 & 0.367 & & -0.2 \\
\hline $1128+385$ & 0.91 & 0.06 & 1 & 0.769 & 0.11 & -0.2 \\
\hline $1137+179$ & 0.25 & 0.03 & 1 & 0.583 & -0.56 & -0.5 \\
\hline $1142+052$ & 0.25 & 0.04 & 1 & 0.486 & -0.44 & -0.2 \\
\hline $1144+402$ & 1.31 & 0.06 & 1 & 0.836 & 0.30 & -0.2 \\
\hline $1144+352$ & 0.18 & 0.04 & 1 & 0.669 & & -0.2 \\
\hline $1147+245$ & 0.74 & 0.01 & 45 & 0.645 & 0.09 & 0.0 \\
\hline $1150+095$ & 0.19 & 0.04 & 1 & 0.432 & & -0.3 \\
\hline $1150+497$ & 1.42 & 0.05 & 33 & 0.717 & 0.45 & -0.6 \\
\hline $1151+408$ & 0.36 & 0.02 & 1 & 0.374 & -0.03 & -0.5 \\
\hline $1154+098$ & 0.02 & 0.02 & 1 & 0.245 & & -0.4 \\
\hline $1155+251$ & 0.21 & 0.04 & 1 & 1.161 & -1.13 & 0.1 \\
\hline $1155+169$ & 0.53 & 0.06 & 1 & 0.870 & -0.33 & 0.3 \\
\hline $1156+295 *$ & 1.97 & 0.05 & 183 & 1.461 & 0.20 & -0.1 \\
\hline $1204+281$ & 0.27 & 0.04 & 1 & 0.457 & -0.35 & -0.1 \\
\hline $1210+133$ & 0.04 & 0.05 & 1 & 0.894 & & -0.4 \\
\hline $1211+334$ & 0.10 & 0.03 & 1 & 0.627 & & -0.5 \\
\hline $1212+177$ & 0.02 & 0.04 & 1 & 0.501 & & -0.3 \\
\hline $1213+350$ & 0.58 & 0.06 & 1 & 1.123 & -0.44 & -0.3 \\
\hline $1215+303$ & 0.40 & 0.02 & 27 & 0.478 & -0.12 & -0.1 \\
\hline $1216+487$ & 0.76 & 0.15 & 2 & 0.638 & 0.12 & -0.2 \\
\hline $1216+061$ & 1.24 & 0.07 & 1 & 4.044 & -0.78 & -1.3 \\
\hline $1217+023$ & 0.43 & 0.05 & 4 & 0.650 & -0.27 & 0.0 \\
\hline $1217+295$ & 0.17 & 0.04 & 1 & 0.372 & & -0.4 \\
\hline $1218+444$ & 0.18 & 0.05 & 1 & 0.539 & & -0.2 \\
\hline
\end{tabular}


Table 1. continued

\begin{tabular}{|c|c|c|c|c|c|c|c|c|c|c|c|c|c|}
\hline Source & $S(22)$ & $\sigma$ & $n$ & $S(5)$ & $\alpha_{2}$ & $\alpha_{1}$ & Source & $S(22)$ & $\sigma$ & $n$ & $S(5)$ & $\alpha_{2}$ & $\alpha_{1}$ \\
\hline $1219+044$ & 1.99 & 0.04 & 1 & 1.351 & 0.26 & 0.3 & $1424+046$ & 0.04 & 0.08 & 1 & 0.174 & & -0.5 \\
\hline $1222+037$ & 0.91 & 0.10 & 1 & 1.221 & -0.19 & 0.0 & $1427+543$ & 0.50 & 0.04 & 1 & 0.716 & -0.24 & -0.2 \\
\hline $1222+131$ & 1.05 & 0.04 & 1 & 3.168 & -0.73 & -0.5 & $1427+109$ & 0.50 & 0.04 & 1 & 1.194 & -0.58 & 1.3 \\
\hline $1222+216 *$ & 1.68 & 0.04 & 101 & 1.154 & 0.11 & -0.4 & $1432+200$ & 0.28 & 0.09 & 1 & 0.692 & & -0.1 \\
\hline $1223+395$ & 0.25 & 0.08 & 1 & 0.506 & & -0.2 & $1433+177$ & 0.24 & 0.03 & 1 & 0.529 & -0.52 & -0.4 \\
\hline $1223+099$ & 0.13 & 0.08 & 1 & 0.478 & & -0.4 & $1434+235$ & 0.20 & 0.10 & 1 & 0.764 & & 0.0 \\
\hline $1226+023 *$ & 33.99 & 0.49 & 289 & 43.572 & -0.16 & -0.1 & $1435+638$ & 0.72 & 0.06 & 1 & 0.757 & -0.03 & -0.5 \\
\hline $1227+181$ & 0.14 & 0.02 & 1 & 0.419 & -0.72 & -0.5 & $1438+385$ & 0.34 & 0.07 & 1 & 0.886 & & -0.1 \\
\hline $1227+255$ & 0.31 & 0.04 & 1 & 0.324 & -0.03 & -0.4 & $1439+137$ & 0.11 & 0.04 & 1 & 0.331 & & -0.4 \\
\hline $1228+126$ & 21.64 & 0.03 & 316 & 59.027 & -0.66 & 0.8 & $1442+637$ & 0.02 & 0.04 & 1 & 0.442 & & -0.3 \\
\hline $1230+486$ & 0.25 & 0.04 & 1 & 0.268 & -0.05 & -0.4 & $1442+101$ & 0.11 & 0.05 & 1 & 1.275 & & -0.5 \\
\hline $1235+196$ & 0.28 & 0.07 & 1 & 0.459 & & -0.2 & $1444+217$ & 0.15 & 0.05 & 1 & 0.295 & & -0.5 \\
\hline $1238+243$ & 0.12 & 0.04 & 1 & 0.330 & & -0.4 & $1444+175$ & 0.77 & 0.13 & 1 & 0.677 & 0.09 & 0.2 \\
\hline $1239+376$ & 0.31 & 0.07 & 1 & 0.372 & & -0.1 & $1451+270$ & 0.31 & 0.01 & 2 & 0.351 & -0.08 & -0.5 \\
\hline $1240+099$ & 0.09 & 0.05 & 1 & 0.270 & & -0.4 & $1452+165$ & 0.21 & 0.06 & 1 & 0.397 & & -0.5 \\
\hline $1241+735$ & 0.48 & 0.09 & 1 & 0.312 & 0.28 & -0.3 & $1452+301$ & 0.18 & 0.05 & 1 & 0.406 & & -0.5 \\
\hline $1250+532$ & 0.41 & 0.05 & 1 & 0.363 & 0.08 & -0.3 & $456+092$ & 0.11 & 0.06 & 1 & 0.287 & & -0.5 \\
\hline $1252+119$ & 0.62 & 0.04 & 1 & 0.724 & -0.10 & -0.3 & $1456+044$ & 0.79 & 0.12 & 1 & 0.615 & 0.17 & -0.2 \\
\hline $1252+092$ & 0.26 & 0.07 & 1 & 0.395 & & -0.2 & $1458+718$ & 1.40 & 0.05 & 1 & 3.567 & -0.62 & -0.6 \\
\hline $1255+327$ & 0.39 & 0.03 & 1 & 0.488 & -0.15 & 0.0 & $1502+106$ & 1.27 & 0.03 & 89 & 2.325 & -0.40 & 0.3 \\
\hline $1258+507$ & 0.16 & 0.08 & 2 & 0.442 & & -0.2 & $1504+377$ & 0.87 & 0.12 & 4 & 0.974 & -0.07 & -0.1 \\
\hline $1307+121$ & 0.73 & 0.04 & 1 & 1.350 & -0.41 & 0.3 & $1506+591$ & -0.04 & 0.03 & 1 & 0.291 & & -0.5 \\
\hline $1308+326$ & 2.70 & 0.06 & 193 & 1.447 & 0.41 & -0.3 & $1509+022$ & 0.29 & 0.06 & 1 & 0.510 & & -0.5 \\
\hline $1308+145$ & 0.14 & 0.04 & 1 & 0.401 & & -0.4 & $1509+158$ & 0.25 & 0.05 & 1 & 0.434 & -0.36 & -0.4 \\
\hline $1308+172$ & 0.08 & 0.03 & 1 & 0.402 & & -0.3 & $1510+472$ & 0.06 & 0.05 & 2 & 0.390 & & -0.5 \\
\hline $1310+560$ & 0.15 & 0.06 & 1 & 0.268 & & -0.5 & $511+238$ & 0.18 & 0.02 & 1 & 0.794 & -0.98 & -0.5 \\
\hline $1317+199$ & 0.15 & 0.06 & 1 & 0.325 & & -0.5 & $514+004$ & 1.3 & 0.04 & 1 & 1.585 & -0.11 & -0.4 \\
\hline $1317+520$ & 0.10 & 0.08 & 1 & 0.614 & & -0.5 & $1522+112$ & 0.19 & 0.03 & 1 & 0.286 & -0.27 & -0.5 \\
\hline $1322+366$ & 0.38 & 0.05 & 1 & 0.403 & -0.04 & -0.5 & $1525+314$ & 0.18 & 0.08 & 1 & 0.555 & & -0.3 \\
\hline $1323+321$ & 0.85 & 0.14 & 1 & 2.234 & -0.64 & -0.6 & $1527+192$ & 0.08 & 0.02 & 1 & 0.305 & & -0.5 \\
\hline $1325+436$ & 0.27 & 0.08 & 1 & 0.576 & & -0.2 & $1528+060$ & -0.03 & 0.08 & 1 & 0.315 & & -0.5 \\
\hline $1327+321$ & 1.17 & 0.04 & 1 & & 0.54 & -0.1 & $1531+722$ & 0.1 & 0.08 & 1 & 0.444 & & -0.3 \\
\hline $1328+254$ & 1.03 & 0.13 & 1 & 3.289 & -0.77 & -0.7 & $1532+016$ & 0.88 & 0.06 & 2 & 1.285 & -0.25 & 0.0 \\
\hline $1328+307$ & 2.45 & 0.06 & 1 & 6.350 & -0.63 & -0.6 & $1535+004$ & 0.14 & 0.12 & 1 & 0.619 & & 0.0 \\
\hline $1330+022$ & 1.17 & 0.09 & 1 & 1.369 & -0.10 & -0.7 & $1538+082$ & 1.00 & 0.05 & 1 & 0.430 & 0.56 & -0.3 \\
\hline $1333+589$ & 0.36 & 0.05 & 1 & 0.740 & -0.48 & -0.1 & $538+149$ & 0.84 & 0.02 & 142 & 1.210 & -0.24 & -0.2 \\
\hline $1335+552$ & 0.18 & 0.06 & 1 & & & 0.1 & & & 0.05 & 2 & 0.387 & & -0.5 \\
\hline $1337+637$ & 0.20 & 0.22 & 2 & 0.419 & & -0.1 & $1543+005$ & 0.28 & 0.04 & 1 & 1.309 & -1.02 & -0.3 \\
\hline $1342+663$ & 0.48 & 0.24 & 2 & 0.545 & & -0.4 & $1543+480$ & 0.25 & 0.07 & 1 & 0.437 & & -0.3 \\
\hline $1345+125$ & 1.01 & 0.07 & 3 & 3.112 & -0.74 & -0.4 & $1545+497$ & 0.27 & 0.03 & 1 & 0.523 & -0.44 & -0.4 \\
\hline $1347+539$ & 0.60 & 0.06 & 1 & 0.644 & -0.05 & -0.5 & $1546+027$ & 1.30 & 0.16 & 2 & 1.111 & 0.10 & -0.1 \\
\hline $1354+195$ & 1.37 & 0.03 & 2 & 2.618 & -0.43 & 0.0 & $1547+507$ & 0.91 & 0.05 & 1 & 0.731 & 0.14 & 0.1 \\
\hline $1355+441$ & 0.15 & 0.04 & 1 & 0.430 & & -0.4 & $1548+114$ & 0.15 & 0.08 & 1 & 0.543 & & -0.1 \\
\hline $1356+478$ & 0.19 & 0.05 & 1 & 0.492 & & -0.3 & $1548+056$ & 2.52 & 0.05 & 45 & 3.702 & -0.25 & 0.4 \\
\hline $1400+162$ & 0.14 & 0.07 & 1 & & & -0.5 & $1549+703$ & 0.17 & 0.05 & 1 & 0.340 & & -0.5 \\
\hline $1401+092$ & 0.04 & 0.04 & 1 & 0.290 & & -0.4 & $1551+130$ & 0.41 & 0.06 & 1 & 0.742 & -0.39 & -0.2 \\
\hline $1402+044$ & 0.81 & 0.10 & 1 & 1.008 & -0.14 & 0.6 & $1559+140$ & 0.22 & 0.08 & 1 & 0.365 & & -0.5 \\
\hline $1404+286$ & 0.75 & 0.08 & 1 & 2.353 & -0.76 & 0.7 & $1600+335$ & 0.69 & 0.05 & 1 & 1.656 & -0.58 & -0.2 \\
\hline $1409+524$ & 1.04 & 0.13 & 1 & 6.764 & -1.23 & -0.9 & $1602+178$ & 0.02 & 0.04 & 1 & 0.386 & & -0.5 \\
\hline $1413+135$ & 1.87 & 0.06 & 141 & 0.846 & 0.52 & -0.3 & $1604+183$ & 0.17 & 0.03 & 1 & 0.258 & -0.28 & -0.5 \\
\hline $1413+349$ & 0.30 & 0.03 & 1 & 0.888 & -0.72 & -0.5 & $1604+315$ & 0.32 & 0.03 & 1 & 0.453 & -0.23 & -0.5 \\
\hline $1415+463$ & 0.33 & 0.05 & 1 & 0.797 & -0.58 & -0.1 & $1604+159 *$ & 0.60 & 0.05 & 1 & 0.515 & 0.10 & -0.2 \\
\hline $1417+385$ & 1.23 & 0.05 & 1 & 0.651 & 0.42 & 0.2 & $1606+106 *$ & 1.34 & 0.04 & 66 & 1.412 & -0.03 & 0.0 \\
\hline $1418+546$ & 1.01 & 0.04 & 132 & 1.350 & -0.19 & 0.1 & $1611+343 *$ & 3.59 & 0.07 & 129 & 2.324 & 0.29 & -0.1 \\
\hline $1420+326$ & 0.22 & 0.04 & 1 & 0.357 & -0.32 & -0.2 & $1615+364$ & 0.12 & 0.02 & 1 & 0.286 & -0.57 & -0.4 \\
\hline $1421+122$ & 0.18 & 0.09 & 1 & 0.571 & & -0.2 & $1616+366$ & 0.10 & 0.03 & 1 & 0.268 & & -0.5 \\
\hline
\end{tabular}

Table 1. continued 
Table 1. continued

Table 1. continued

\begin{tabular}{|c|c|c|c|c|c|c|c|c|c|c|c|c|c|}
\hline Source & $S(22)$ & $\sigma$ & $n$ & $S(5)$ & $\alpha_{2}$ & $\alpha_{1}$ & Source & $S(22)$ & $\sigma$ & $n$ & $S(5)$ & $\alpha_{2}$ & $\alpha_{1}$ \\
\hline $1616+063$ & 0.32 & 0.09 & 1 & 0.971 & & -0.2 & $1755+578$ & 0.03 & 0.04 & 1 & 0.463 & & -0.4 \\
\hline $1623+578$ & 0.27 & 0.03 & 1 & 0.585 & -0.51 & 0.1 & $1756+237$ & 0.25 & 0.08 & 2 & 0.929 & & 0.3 \\
\hline $1624+416$ & 0.52 & 0.06 & 3 & 1.253 & -0.58 & -0.2 & $1758+666$ & 0.67 & 0.07 & 1 & 0.874 & -0.18 & 0.1 \\
\hline $1625+582$ & 0.20 & 0.05 & 1 & 0.315 & & -0.5 & $1758+388$ & 1.54 & 0.09 & 1 & 0.735 & 0.49 & 0.3 \\
\hline $1629+120$ & 0.14 & 0.03 & 1 & 0.864 & & -0.4 & $1800+440$ & 0.53 & 0.18 & 4 & 1.193 & -0.54 & 0.2 \\
\hline $1633+382 *$ & 2.20 & 0.03 & 201 & 3.221 & -0.25 & 0.4 & $1807+698$ & 1.53 & 0.03 & 100 & 2.122 & -0.22 & 0.0 \\
\hline $1636+473$ & 1.07 & 0.05 & 1 & 1.244 & -0.10 & 0.3 & $1807+279$ & 0.27 & 0.03 & 1 & 0.449 & -0.34 & -0.5 \\
\hline $1636+106$ & 0.12 & 0.05 & 1 & 0.505 & & -0.5 & $1809+568$ & 0.69 & 0.08 & 1 & 0.569 & 0.13 & 0.0 \\
\hline $1637+574$ & 1.56 & 0.05 & 104 & 1.750 & -0.08 & 0.3 & $1811+430$ & 0.60 & 0.05 & 1 & 0.510 & 0.11 & -0.5 \\
\hline $1638+124$ & 0.32 & 0.04 & 1 & 1.207 & -0.88 & -0.4 & $1812+412$ & 0.26 & 0.05 & 1 & 0.517 & -0.45 & -0.2 \\
\hline $1638+398$ & 1.15 & 0.06 & 14 & 1.117 & 0.02 & 0.5 & $1814+349$ & 0.08 & 0.04 & 1 & 0.338 & & -0.5 \\
\hline $1640+254$ & 1.00 & 0.12 & 1 & 0.515 & 0.44 & -0.1 & $1815+614$ & 0.05 & 0.13 & 2 & 0.460 & & -0.5 \\
\hline $1641+399$ & 9.40 & 0.17 & 269 & 8.719 & 0.05 & 0.1 & $1818+356$ & 0.30 & 0.08 & 1 & 0.564 & & -0.4 \\
\hline $1641+074$ & 0.20 & 0.02 & 1 & 0.313 & -0.30 & -0.4 & $1823+568$ & 1.85 & 0.09 & 40 & 1.263 & 0.25 & -0.2 \\
\hline $1642+053$ & 0.08 & 0.10 & 1 & 0.363 & & -0.4 & $1828+487$ & 2.87 & 0.04 & 55 & 5.519 & -0.43 & -0.9 \\
\hline $1642+690$ & 1.01 & 0.05 & 19 & 1.527 & -0.27 & 0.0 & $1842+681$ & 0.44 & 0.08 & 1 & 0.925 & -0.49 & 0.3 \\
\hline $1642+256$ & 0.09 & 0.06 & 1 & 0.400 & & -0.5 & $1849+670$ & 0.73 & 0.08 & 1 & 0.845 & -0.10 & 0.1 \\
\hline $1645+271$ & 0.25 & 0.07 & 1 & 0.284 & & -0.5 & $1856+737$ & 0.54 & 0.04 & 1 & 0.576 & -0.04 & 0.1 \\
\hline $1648+015$ & 0.51 & 0.07 & 1 & 0.505 & 0.01 & -0.2 & $1928+738$ & 3.28 & 0.03 & 99 & 3.626 & -0.07 & 0.0 \\
\hline $1648+050$ & 1.80 & 0.15 & 1 & 11.596 & -1.23 & -1.1 & $1928+681$ & 0.15 & 0.03 & 1 & 0.306 & -0.47 & -0.4 \\
\hline $1652+398$ & 1.07 & 0.01 & 134 & 1.375 & -0.17 & 0.0 & $1936+714$ & 0.73 & 0.07 & 1 & 0.398 & 0.40 & -0.2 \\
\hline $1655+077$ & 1.29 & 0.12 & 3 & 1.641 & -0.16 & 0.0 & $1946+708$ & 0.25 & 0.08 & 2 & 0.677 & & -0.3 \\
\hline $1656+348$ & 0.24 & 0.10 & 1 & 0.474 & & -0.1 & $2010+723$ & 0.80 & 0.09 & 1 & 0.907 & -0.08 & -0.2 \\
\hline $1656+477$ & 1.07 & 0.05 & 1 & 1.244 & -0.10 & 0.5 & $2059+034$ & 1.24 & 0.04 & 1 & 1.362 & -0.06 & 0.7 \\
\hline $1656+482$ & 0.54 & 0.08 & 1 & 0.738 & -0.21 & -0.1 & $2103+124$ & 0.48 & 0.06 & 1 & 0.656 & -0.21 & -0.5 \\
\hline $1656+571$ & 0.41 & 0.05 & 1 & 0.764 & -0.41 & 0.0 & $2115+056$ & 0.13 & 0.06 & 1 & 0.280 & & -0.4 \\
\hline $1656+053$ & 0.40 & 0.07 & 3 & 1.382 & -0.82 & 0.0 & $2118+188$ & 0.41 & 0.04 & 1 & 0.425 & -0.02 & -0.2 \\
\hline $1658+302$ & 0.13 & 0.03 & 1 & 0.307 & & -0.5 & $2120+099$ & 0.28 & 0.05 & 1 & 0.527 & -0.42 & -0.4 \\
\hline $1706+006$ & 0.48 & 0.06 & 2 & 0.396 & 0.13 & -0.4 & $2121+053$ & 1.90 & 0.15 & 39 & 2.784 & -0.25 & 0.8 \\
\hline $1706+094$ & 0.11 & 0.05 & 1 & 0.326 & & -0.4 & $2128+048$ & 0.35 & 0.09 & 1 & 2.193 & & -0.7 \\
\hline $1708+006$ & 0.13 & 0.04 & 1 & 0.637 & & -0.5 & $2134+004$ & 4.97 & 0.04 & 147 & 10.938 & -0.52 & 0.8 \\
\hline $1713+218$ & 0.08 & 0.06 & 1 & 0.703 & & -0.5 & $2136+141$ & 1.52 & 0.04 & 49 & 1.073 & 0.23 & -0.2 \\
\hline $1714+219$ & 0.30 & 0.09 & 1 & 0.703 & & 0.1 & $2141+175$ & 1.11 & 0.05 & 26 & 1.006 & 0.07 & 0.7 \\
\hline $1716+006$ & 0.16 & 0.03 & 1 & 0.987 & -1.20 & -0.5 & $2144+092$ & 0.75 & 0.03 & 43 & 1.233 & -0.33 & 0.3 \\
\hline $1717+178$ & 0.99 & 0.26 & 3 & 0.559 & 0.38 & -0.3 & $2145+067$ & 8.33 & 0.10 & 204 & 4.135 & 0.46 & 0.3 \\
\hline $1719+357$ & -0.05 & 0.05 & 1 & 0.784 & & 0.0 & $2147+077$ & 0.16 & 0.06 & 1 & 0.420 & & -0.3 \\
\hline $1720+250$ & 0.03 & 0.03 & 1 & 0.378 & & -0.5 & $2149+069$ & 0.78 & 0.08 & 1 & 0.794 & -0.01 & 0.1 \\
\hline $1722+526$ & 0.34 & 0.04 & 1 & 0.334 & 0.01 & -0.5 & $2149+056$ & 0.51 & 0.05 & 1 & 0.892 & -0.37 & 0.1 \\
\hline $1722+401$ & 0.73 & 0.09 & 1 & 0.524 & 0.22 & 0.0 & $2150+173$ & 0.65 & 0.05 & 3 & 0.757 & -0.10 & -0.2 \\
\hline $1724+399$ & 0.05 & 0.03 & 1 & 0.296 & & -0.5 & $2152+226$ & 0.27 & 0.05 & 1 & 0.505 & -0.41 & -0.1 \\
\hline $1725+123$ & 0.44 & 0.08 & 1 & 0.881 & -0.46 & 0.2 & $2201+171$ & 1.79 & 0.07 & 1 & 0.834 & 0.51 & -0.2 \\
\hline $1725+044 *$ & 0.78 & 0.03 & 63 & 0.885 & -0.08 & 0.3 & $2201+044$ & 0.40 & 0.05 & 1 & 0.653 & -0.32 & 0.0 \\
\hline $1732+094$ & 0.47 & 0.06 & 1 & 0.897 & -0.43 & 0.0 & $2209+236 *$ & 1.15 & 0.05 & 1 & 1.123 & 0.02 & 0.7 \\
\hline $1732+389$ & 0.57 & 0.07 & 1 & 0.557 & 0.02 & -0.3 & $2214+307$ & 0.24 & 0.07 & 2 & 0.531 & & -0.3 \\
\hline $1734+508$ & 0.66 & 0.05 & 1 & 0.755 & -0.09 & 0.1 & $2215+020$ & 0.25 & 0.04 & 1 & 0.513 & -0.48 & -0.3 \\
\hline $1738+499$ & 1.14 & 0.06 & 1 & 0.428 & 0.65 & -0.2 & $2223+210$ & 0.73 & 0.09 & 1 & 1.024 & -0.22 & -0.4 \\
\hline $1738+476$ & 0.34 & 0.09 & 1 & 0.818 & & 0.0 & $2224+006$ & 0.44 & 0.06 & 1 & 0.473 & -0.05 & -0.2 \\
\hline $1739+522 *$ & 1.81 & 0.06 & 100 & 1.699 & 0.04 & -0.4 & $2226+325$ & 0.15 & 0.06 & 1 & 0.295 & & -0.5 \\
\hline $1741+196$ & 0.21 & 0.04 & 1 & 0.353 & -0.34 & -0.4 & $2230+114 *$ & 3.51 & 0.10 & 163 & 3.967 & -0.08 & -0.5 \\
\hline $1743+173$ & 0.70 & 0.08 & 1 & 0.690 & 0.01 & -0.4 & $2234+202$ & 0.09 & 0.05 & 1 & 0.363 & & -0.5 \\
\hline $1743+557$ & 0.55 & 0.07 & 1 & 0.562 & -0.01 & -0.2 & $2234+282$ & 1.04 & 0.04 & 42 & 1.625 & -0.30 & 0.5 \\
\hline $1745+624$ & 0.31 & 0.07 & 1 & 0.589 & & -0.2 & $2245+029$ & 0.53 & 0.07 & 1 & 0.588 & -0.07 & -0.5 \\
\hline $1745+670$ & 0.02 & 0.06 & 1 & 0.288 & & -0.5 & $2246+208$ & 0.82 & 0.08 & 1 & 0.724 & 0.08 & 0.1 \\
\hline $1749+701$ & 0.62 & 0.06 & 25 & 0.715 & -0.09 & -0.5 & $2247+140$ & 0.58 & 0.06 & 1 & 1.240 & -0.50 & -0.5 \\
\hline $1751+288$ & 0.51 & 0.06 & 1 & 0.511 & 0.00 & -0.2 & $2247+113$ & 0.46 & 0.03 & 1 & 0.994 & -0.51 & -0.4 \\
\hline $1751+441$ & 0.65 & 0.11 & 2 & 1.000 & -0.28 & 0.2 & $2251+134$ & 0.65 & 0.10 & 1 & 0.877 & -0.20 & -0.5 \\
\hline
\end{tabular}


Table 1. continued

\begin{tabular}{lrlllrr}
\hline Source & $S(22)$ & $\sigma$ & $n$ & $S(5)$ & $\alpha_{2}$ & $\alpha_{1}$ \\
\hline $2251+158 *$ & 9.56 & 0.14 & 266 & 14.468 & -0.27 & 0.1 \\
$2251+006$ & 0.35 & 0.10 & 1 & 0.419 & & -0.1 \\
$2259+371$ & 0.55 & 0.09 & 1 & 0.442 & 0.14 & -0.3 \\
$2301+060$ & 0.35 & 0.08 & 1 & 0.561 & & -0.1 \\
$2304+322$ & 0.32 & 0.04 & 1 & 0.468 & -0.25 & -0.4 \\
$2307+106$ & 0.36 & 0.05 & 1 & 0.504 & -0.22 & -0.3 \\
$2308+341$ & 0.54 & 0.07 & 1 & 0.994 & -0.40 & 0.0 \\
$2310+385$ & 0.26 & 0.07 & 1 & 0.532 & & -0.3 \\
$2314+160$ & -0.01 & 0.07 & 1 & 0.403 & & -0.3 \\
$2318+049$ & 0.99 & 0.05 & 2 & 1.048 & -0.04 & 0.3 \\
$2319+272$ & 0.53 & 0.01 & 4 & 0.962 & -0.39 & -0.1 \\
$2320+079$ & 0.75 & 0.09 & 1 & 0.857 & -0.09 & -0.2 \\
$2325+093$ & 1.08 & 0.09 & 1 & 0.643 & 0.34 & 0.1 \\
$2328+107$ & 0.63 & 0.05 & 2 & 1.194 & -0.42 & 0.1 \\
$2328+316$ & 0.21 & 0.04 & 1 & 0.557 & -0.65 & -0.5 \\
$2331+073$ & 1.37 & 0.05 & 1 & 1.374 & 0.00 & 0.3 \\
$2337+264$ & 0.41 & 0.08 & 1 & 0.890 & -0.51 & -0.1 \\
$2344+092$ & 1.12 & 0.03 & 38 & 1.392 & -0.14 & -0.2 \\
$2345+061$ & 0.19 & 0.04 & 1 & 0.343 & & -0.4 \\
$2356+196 *$ & 0.28 & 0.06 & 1 & 0.708 & & 0.2 \\
$2356+385$ & 0.43 & 0.05 & 1 & 0.479 & -0.07 & -0.3 \\
$2358+406$ & 0.15 & 0.03 & 1 & 0.595 & -0.91 & -0.5 \\
\hline & & & & & &
\end{tabular}

high noise from the receiver (300 K DSB) and the radome attenuation, this can not be fully utilized. In some cases a few observations have been done years apart and even all the individual observations have been with a fair signal to noise ratio $(\geq 5)$; the error calculated from the mean value has been larger than 0.2 times the mean value, due to the variations in the source. In these cases the spectral index has been given. A plot of spectral indexes for all observed sources in the selected sample between 22 and $4.85 \mathrm{GHz}$ $\left(\alpha_{\mathrm{hi}}\right)$ and 4.85 and $1.4 \mathrm{GHz}\left(\alpha_{\mathrm{lo}}\right)$ is shown in Fig. 1 . It is clear, that when going to lower spectral indexes (between 1.4 and $4.85 \mathrm{GHz}$ ), towards steeper spectra, the sources in general are also weaker at $22 \mathrm{GHz}$. Thus a greater fraction of sources remain undetected when observing at $22 \mathrm{GHz}$. The number of sources detected at $22 \mathrm{GHz}$ with different spectral indexes $\left(\alpha_{\mathrm{lo}}\right)$ and the fraction of them being flat spectrum $\left(\alpha_{\mathrm{hi}} \geq-0.3\right)$ sources can be sen in Table 2 . In Fig. 1 the individual $\alpha_{2}-\alpha_{1}$ pairs are marked with triangles, while sources which have also been detected at $\gamma$-rays have been marked with a circle.

Clearly the $\gamma$-detected sources are more compactly in the $\alpha_{\text {hi }}$ than in the $\alpha_{\text {lo }}$ space. This may be used as an indication that the $\gamma$-radiation is better connected to the mm-radiation rather than to the cm-radiation of AGN. When simply calculating the mean values of the spectral indexes for $\gamma$-ray detected sources, both high and low frequency, we get $\alpha_{\mathrm{hi}}=-0.03 \pm 0.03$ and $\alpha_{\mathrm{lo}}=0.03 \pm 0.06$. When looking at the spread of the $\alpha_{\text {lo }}$ for the $\gamma$-detected sources, one notes that there are sources from all three types, steep spectrum, flat spectrum and inverted spectrum, as the same sources in the $\alpha_{\text {hi }}$ plane can all be classified as flat spectrum sources $\left|\alpha_{\mathrm{hi}}\right| \leq 0.3$.
Table 2. Number of sources detected at $22 \mathrm{GHz}$ with different spectral indexes $\left(\alpha_{\mathrm{lo}}\right)$, total number of sources, detection fraction $\left(\delta_{\mathrm{d}}\right)$, number of sources having flat spectrum $\left(\alpha_{\mathrm{hi}} \geq-0.3\right)$ and their fraction from detected sources $\left(\delta_{\text {flat }}\right)$

\begin{tabular}{rrrlrl}
\hline$\alpha_{\text {lo }}$ & detected & total & $\delta_{\mathrm{d}}$ & $\alpha_{\mathrm{hi}} \geq-0.3$ & $\delta_{\text {flat }}$ \\
\hline-0.5 & 35 & 93 & 0.38 & 19 & 0.54 \\
-0.4 & 38 & 65 & 0.58 & 19 & 0.50 \\
-0.3 & 25 & 44 & 0.57 & 19 & 0.76 \\
-0.2 & 43 & 60 & 0.72 & 35 & 0.81 \\
-0.1 & 38 & 48 & 0.79 & 32 & 0.84 \\
0.0 & 37 & 44 & 0.84 & 29 & 0.78 \\
0.1 & 24 & 28 & 0.96 & 19 & 0.79 \\
0.2 & 18 & 20 & 0.90 & 16 & 0.89 \\
0.3 & 17 & 18 & 0.94 & 11 & 0.65 \\
$\geq 0.4$ & 28 & 28 & 1.00 & 19 & 0.68 \\
\hline
\end{tabular}

The expansion of this study to even lower flux levels $(S<0.5 \mathrm{Jy}$ at $1.4 \mathrm{GHz})$ and steeper spectrum sources would leave more than half of the sources undetected and would serve mainly as a detector of inverted spectrum sources or flat spectrum sources when in a high state. If we were to put the lower limit in $1.4 \mathrm{GHz}$ flux to $0.2 \mathrm{Jy}$, we would have 456 new sources to observe, in the group with $\alpha>-0.3$ and respectively 135 for $\alpha=-0.3,161$ for $\alpha=-0.4$ and 272 for $\alpha=-0.5$.

The extended sources, which had a flux given at 1.4 and $0.365 \mathrm{GHz}$, but not at $4.85 \mathrm{GHz}$, should be observed also at $22 \mathrm{GHz}$. The number of such sources fulfilling our other criteria is 21 . The number of sources with no 0.365 and $4.85 \mathrm{GHz}$ flux is about 100 . A more sensitive receiving system would be needed to deepen this study as some of the sources currently in our monitoring sample, like III ZW 2 and $0109+224$ have flux densities in the $1.4 \mathrm{GHz}$ cataloque in the range of $0.3 \mathrm{Jy}$, but can reach $3 \mathrm{Jy}$ at $22 \mathrm{GHz}$ during outburst stage.

\section{Predictions for source counts}

Though the sample in this study is not complete for the extragalactic sources, as the extended sources from the $1.4 \mathrm{GHz}$ cataloque, which were not given a spectral index, have been neclected, we can still try to estimate the number of sources on the Northern sky having the $22 \mathrm{GHz}$ flux on average over a certain flux limit. We can also estimate the number of sources reaching certain flux limits, while at their maximum, based on our 20 years monitoring records. For these purposes we need to make a simple loglog plot on the number of sources and limiting flux density. First we calculate the sources exceeding 5, 2 and 1 Jy on average. Our sample is not quite complete to the $1 \mathrm{Jy}$ limit, not even to the $2 \mathrm{Jy}$ limit and the stronger sources $(S \geq 5$ Jy) are very few. Calculating the slope for the three $\log (N) / \log (S)$ points gives a value of $\beta=1.76$, according to $N(>S) \propto S^{-\beta}$, which is the same as calculated from the source counts in Gregory et al. (1996), taking into account only the sources over 5,2 and 1 Jy. If we make a 


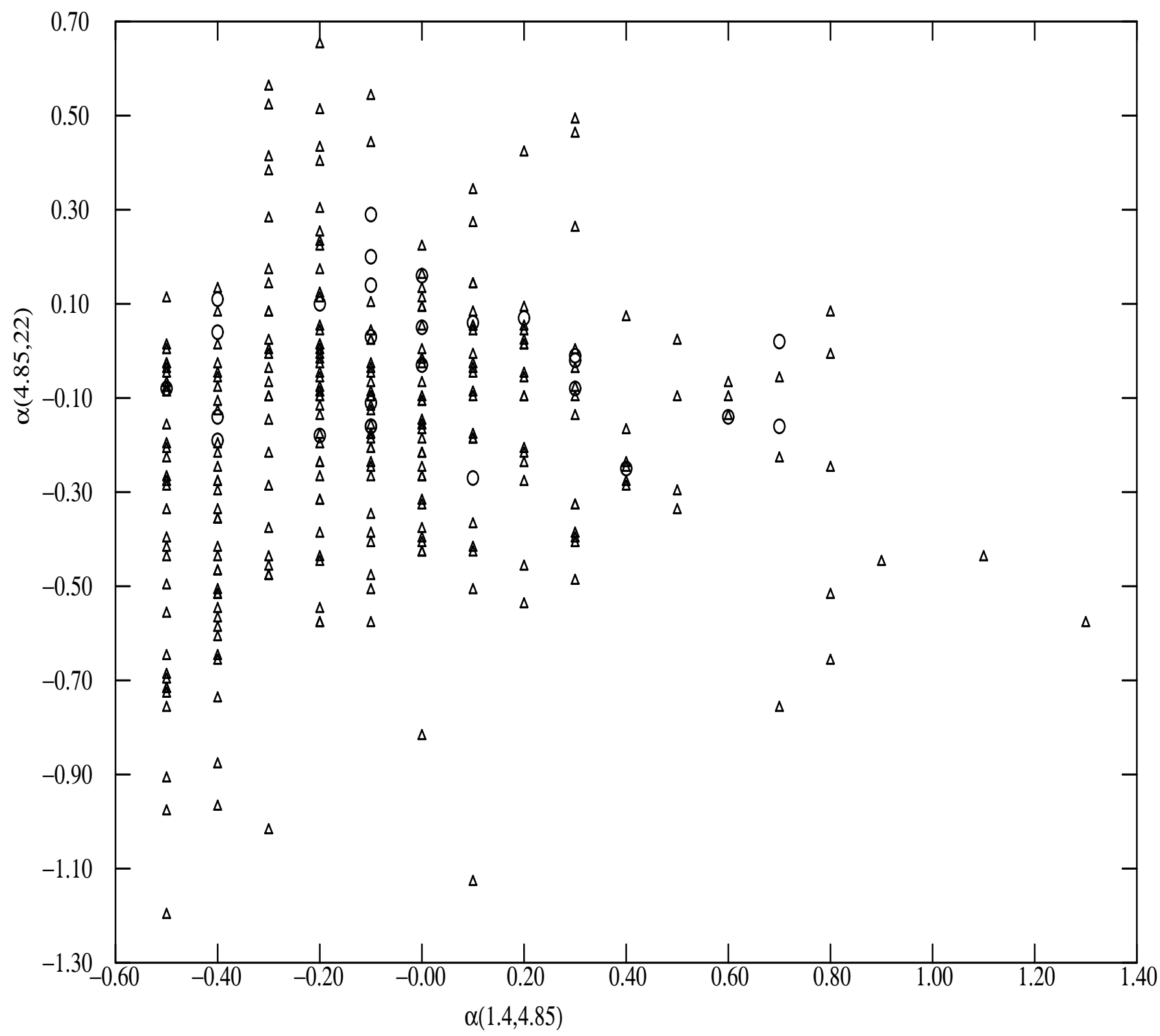

Fig. 1. Plot of the spectral indexes $\alpha_{\mathrm{hi}}(4.85$ to $22 \mathrm{GHz})$ vs. $\alpha_{\text {lo }}(1.4$ to $4.85 \mathrm{GHz})$ of the detected sources. Sources detected at $\gamma$-rays have been marked with a circle

similar fitting to the flat spectrum sources, $\alpha_{\mathrm{hi}} \geq-0.3$, we still get the same value $\beta=1.76$ for those same flux limits; even the number of sources is somewhat smaller, as some stronger radio galaxies are excluded. Last we calculate from our monitoring data the number of sources whose maximum fluxes are over 2, 5 and $10 \mathrm{Jy}$. The results of these source counts can be found in Table 3. For both source counts only sources with $|b| \geq 20^{\circ}$ were calculated. By a simple assumption, that the source populations at lower flux levels are similar in this sample than in the original survey (GB6), we would have about 320 sources with an average flux over $0.5 \mathrm{Jy}$ at $22 \mathrm{GHz}$ and about 260 of those would be flat spectrum sources $\left(\alpha_{\text {hi }} \geq-0.3\right)$ within our declination range, $0-+75$ degrees and $|b| \geq 20^{\circ}$. Extrapolating the former to the whole sky would give 655 sources with average flux over $0.5 \mathrm{Jy}$ with
540 of them being flat spectrum sources $\left(\alpha_{\mathrm{hi}} \geq-0.3\right)$. The behaviour of the slope described above down to below mJy levels at $1.4 \mathrm{GHz}$ can be seen in Condon et al. (1998).

All those estimations are to be seen as lower limits, as a few sources for which the mean flux is over $1.0 \mathrm{Jy}$ at $22 \mathrm{GHz}$ should still be found. If we assume that the source populations at 22 and $4.85 \mathrm{GHz}$ remain the same down to $0.1 \mathrm{Jy}$ limit, we would have about 6700 sources over that limit on average and 5500 of them would be flat spectrum sources $\left(\alpha_{\mathrm{hi}} \geq-0.3\right)$, while looking at sources with $|b| \geq 20^{\circ}$. While looking to the distribution from maximum flux levels, even the number of sources is very limited and surely not even close to complete to the $2 \mathrm{Jy}$ level; one notes that the number of sources is about double when comparing to the mean values or to the flat spectrum sources. If we were to try to estimate the number of 
Table 3. Number of sources exceeding certain flux levels from the GB6 cataloque at $5 \mathrm{GHz}\left(N_{\mathrm{GB} 6}\right)$, from the mean fluxes of this study at $22 \mathrm{GHz}\left(N_{\text {mean }}(22)\right)$, from flat spectrum sources of this study $\left(N_{\text {flat }}(22)\right)$ and from maximum flux levels $\left(N_{\max }(22)\right)$

\begin{tabular}{lllll}
\hline$S(\mathrm{Jy})$ & $N_{\text {GB6 }}$ & $N_{\text {mean }}(22)$ & $N_{\text {flat }}(22)$ & $N_{\max }(22)$ \\
\hline 10 & & & & 7 \\
5 & 12 & 6 & 5 & 14 \\
2 & 54 & 22 & 18 & 39 \\
1 & 213 & 100 & 83 & \\
0.5 & 673 & & & \\
0.2 & 2576 & & & \\
0.1 & 6854 & & & \\
\hline
\end{tabular}

potential sources exceeding certain flux levels at $22 \mathrm{GHz}$ in the high state, we should multiply the number estimates from the mean fluxes by roughly 2 .

For the success of the PLANCK cosmic microwave surveyer it is important to be able to extract the effects of strong foreground sources from the maps. The PLANCK frequencies start from $30 \mathrm{GHz}$ and thus the lower frequency surveys cannot be applied directly. The stronger foreground sources can be detected from the maps using isotropic wavelets, Cayon et al. (2000). They have detected all simulated sources above $0.36 \mathrm{Jy}$ level at $100 \mathrm{GHz}$ with a $S / N>4$. To get advantage from the foreground studies one should know the interfering sources weaker than the PLANCK detection limits. This would mean observing sources in excess of 10000 , if going below the $100 \mathrm{mJy}$ level. Most of these sources are nonvariate, at least at the lowest frequencies, and thus a single observation would be adequate for those. A sensitive higher frequency (20$40 \mathrm{GHz}$ ) full sky survey would be needed. The new $\gamma$-ray observatories, AGILE and GLAST will detect hundreds, even thousands of flat spectrum sources prior the flight of PLANCK and will thus be of great help for the foreground studies.

\section{Conclusions}

New flat spectrum radio sources are found for use with the ground support for both the future $\gamma$-ray missions AGILE and GLAST, and the cosmic background radiation observer PLANCK. The observed sources were selected from lower frequency surveys at 1.4 and $4.85 \mathrm{GHz}$ with the following criteria: $\alpha_{1.4-4.85}$ being $\geq-0.5$, the $S \geq 0.5 \mathrm{Jy}$ at $1.4 \mathrm{GHz}$ and distance more than $20^{\circ}$ from the galactic plane. The sensitivity of the Metsähovi receiving system limits the expansion of this study to much weaker sources and similarily to sources with a steeper spectral index, as in the group of sources with the $\alpha_{\text {lo }}=-0.5$ only $38 \%$ were detected with a $S / N \geq 5$. Overall from the detected sources 218 had $\alpha_{\text {hi }} \geq-0.3$.

Acknowledgements. This work has been supported by the Wihuri foundation and the Academy of Finland.

\section{References}

Browne, I. W. A., Patnaik, A. R., Wilkinson, P. N., \& Wrobel, J. M. 1998, MNRAS, 293, 257.

Cayon, L., Sanz, J. L., Barreiro, R. B., et al. 2000, MNRAS, $315,757$.

Condon, J. J., Cotten, W. D., Greisen, E. W., et al. 1998, AJ, 115, 1693.

Gregory, P. C., Scott, W. K., Douglas, K., \& Condon, J. J. 1996, ApJS, 103, 427.

Hartman, R. C., Bertsch, D. L., Bloom, S. D., et al. 1999, ApJS, 123, 79 .

Kovalev, Y. Y., Nizhelsky, N. A., Kovalev, Yu. A., et al. 1999, A\&AS, 139, 545.

Patnaik, A. R., Browne, I. W. A., Wilkinson, P. N., \& Wrobel, J. M. 1992, MNRAS, 254, 655.

Teräsranta, H., Tornikoski, M., Mujunen, A., et al. 1998, A\&AS, 132, 305.

White, R. L., \& Becker, R. H. 1992, ApJS, 79, 331.

Wilkinson, P. N., Browne, I. W. A., Patnaik, A. R., et al. 1998, MNRAS, 300, 790 .

Wiren, S., Valtaoja, E., Teräsranta, H., \& Kotilainen, J. 1992, AJ, 104, 1009. 